\title{
Multi-Model Investigation and Adaptive Estimation of the Acoustic Release of a Model Drug From Liposomes
}

\author{
Ali Wadi ${ }^{\circledR}$, Mamoun Abdel-Hafez ${ }^{\circledR}$, Ghaleb A. Husseini ${ }^{\circledR}$, and Vinod Paul
}

\begin{abstract}
This paper researches a suitable mathematical model that can reliably predict the release of a model drug (namely calcein) from biologically targeted liposomal nanocarriers triggered by ultrasound. Using mathematical models, curve fitting is performed on a set of five experimental acoustic drug release runs from Albumin-, Estrone-, and RGD-based Drug Delivery Systems (DDS). The three moieties were chosen to target specific cancers using receptormediated endocytosis. The best-fitting mathematical model is then enhanced using a Kalman filtering (KF) algorithm to account for the statistics of the dynamic and measurements noise sequences in predicted drug release. Unbiased drug-release estimates are realized by implementing an online noise identification algorithm. The algorithm is first deployed in a simulated environment in which it was rigorously tested and compared with the correct solution. Then, the algorithm was used to process the five experimental datasets. The results suggest that the Adaptive Kalman Filter (AKF) is exceptionally good at handling drug release estimation problems with a priori unknown or with changing noise covariances. In comparison with the KF, the AKF approach exhibited as low as a $69 \%$ reduction in the level of error in estimating the drug release state. Finally, the proposed algorithm is not computationally demanding and is capable of online estimation tasks.
\end{abstract}

Index Terms-Chemotherapy, calcein, drug release, online estimation, adaptive noise identification, adaptive Kalman filter, modeling, liposomes, ultrasound.

\section{INTRODUCTION}

$\mathbf{T}$ HE need to mitigate the adverse side effects of chemotherapeutic treatments has brought about the development of new ways through which these drugs can be administered. The use of Drug Delivery Systems (DDS) in cancer treatment is a trend enabled by nanotechnology; whereby nanoparticles, in the size range of $1 \mathrm{~nm}$ to $1 \mu \mathrm{m}$, are used as chemotherapeutic drug carriers. These nanocarriers encapsulate therapeutic drugs allowing the controlled administration of cytotoxic drugs like alkylators, topoisomerase inhibitors,

Manuscript received October 3, 2018; revised January 21, 2019, May 5, 2019, October 9, 2019, and October 26, 2019; accepted October 26, 2019. Date of publication November 6, 2019; date of current version December 31, 2019. (Corresponding author: Ghaleb A. Husseini.)

A. Wadi and M. Abdel-Hafez are with the Department of Mechanical Engineering, American University of Sharjah, Sharjah, United Arab Emirates (e-mail: awadi@aus.edu; mabdelhafez@aus.edu).

G. A. Husseini and V. Paul are with the Department of Chemical Engineering, American University of Sharjah, Sharjah, United Arab Emirates (e-mail: ghusseini@aus.edu; vpaul@aus.edu).

Digital Object Identifier 10.1109/TNB.2019.2950344 antimetabolites, microtubule, and amino acid depletion agents [1]-[3]. Nanocarriers must release their encapsulated molecules in a controlled manner in a given period. Consequently, modeling the triggered release of chemotherapeutics is beneficial to the deployment of DDS. An effective DDS that uses nanocarriers enables the controlled targeting of cancer cells, thus limiting the healthy cell exposure to the cytotoxic therapeutic agents, and enhancing the quality of life of a cancer patient.

In our lab, we study the multimodal delivery of nanocarriers using three targeting techniques: passive (targeting by optimal size ranging between 15 and $200 \mathrm{~nm}$ ), active (targeting using a ligand/moiety) and acoustically triggered (using ultrasound as the trigger). The nanocarriers, studied in this work, are liposomes; vesicles composed of a phosopholipid bilayer which surrounds an aqueous core. Liposomes can encapsulate hydrophilic drugs in their inner aqueous interior, and hydrophobic drugs within the lipid bilayer. Alternatively, they can be modified with ligand or moiety to specifically target receptors overexpressed on the membrane of cancer cells [4], including albumin [5], estrogen [6], and RGD [7]. Each moiety is used to target certain cancers. The release of chemotherapeutics from any of the above-targeted vehicles may be acoustically-triggered using ultrasound (US). These waves allow the drug load, encapsulated inside these nanocarriers, to target the malignant cells where US is focused, thus sparing the healthy unsonicated cells from the action of the anti-neoplastic agents.

Modeling drug release of chemotherapeutics from DDS has been reported in the literature [8], [9]. Many methodologies are applied to realize mathematical models that have the capacity to predict and quantify the release of the drug from the carrier into the surrounding environment. These methods utilize regression-based statistical approaches, model-dependent approaches, model-independent approaches, and, more recently, nonlinear observers, stochastic estimators, evolutionary algorithms, and other information processing paradigms such as Artificial Neural Networks [9], [10], [19], [11]-[18]. Given the difficulty in identifying the parameters in biological models, other methods that couple the process of state and parameter estimation into one and/or apply constrained optimization on the parameter set to ensure finding physically meaningful parameters have been proposed [20], [21]. Here, five model-dependent kinetic mathematical 
models are investigated to predict the acoustic release of a model drug, namely calcein, from liposomes.

As is the case with all dynamic systems, dynamics and measurements noises affect the process and the measurement apparatus. The former type of noise can be attributed to the uncertainty in the ability of the mathematical model to accurately predict the state of a dynamic system, while the latter is attributed to the uncertainty in the equipment (used to measure drug release) in obtaining an accurate estimate of the dynamic system state [22]. Accounting for the two noise types can prove advantageous to the prediction of drug release. This is achieved by applying a Kalman filter to predict the drug release using the mathematical model and subsequently update that state using the information acquired through available measurements. This is accomplished while accounting for the dynamics and measurements noises plaguing the process [23], [24]. To have the best possible estimate of the drug release state, the uncertainty structure of the noises affecting the process and measurement equations are to be identified, which is achieved by applying one of several algorithms reported in literature including Maximum Likelihood, Autocovariance Least Squares, and Covariance Matching [25]-[27].

Accurate and precise delivery of chemotherapeutics to cancer patients using our proposed DD technique is highly dependent on the estimation algorithm, which gives better estimates of the released drug amounts over mere measurements. This is especially true when and where the measurements are not available for a specified duration, even if this duration is short. An advantage over other statistical approaches, the proposed estimation algorithm can predict the released drug amounts in real-time to successfully maintain the operation of the controlled drug delivery system.

\section{Methods and Mathematical Modelling}

\section{A. Drug Synthesis}

Here, the synthesis of the targeted liposomes is presented. More details can be found in [28]-[30]. Multiple batches for each of the drugs were prepared, and the acoustic release (at $20 \mathrm{kHz}$ ) was measured using a fluorometer.

A similar protocol for preparing albumin and RGD conjugated liposomes was followed. The liposomes were prepared according to the modified lipid film hydration method described by Lasch et al. The lipids: DPPC and DSPE-PEG(2000)- $\mathrm{NH}_{2}$, in addition to the cholesterol, were dissolved in chloroform at a molar ratio of $65: 5: 30$ in a round bottom flask. The chloroform was then evaporated under a reduced pressure using a rotatory evaporator maintained at $50^{\circ} \mathrm{C}$, leading to the formation of a thin lipid film inside the flask. The film was then hydrated with $2 \mathrm{~mL}$ of $50 \mathrm{mM}$ calcein (dissolved in phosphate buffer saline (PBS) and the $\mathrm{pH}$ adjusted to 7.4) using the rotatory evaporator for 50 mins at $60^{\circ} \mathrm{C}$ followed by sonication at $60^{\circ} \mathrm{C}$ using a sonication bath (Agar Scientific) for 2 mins. The formed liposomes were then extruded at $60^{\circ} \mathrm{C}$ through the $0.2-\mu \mathrm{m}$ polycarbonate membrane using Avanti $\AA$ mini-extruder (Avanti Polar Lipids, Inc., Alabaster, AL, USA). The liposomes were purified using Sephadex ${ }^{\circledR}$ G-100 gel filtration (size exclusion chromatography) equilibrated with Borate buffer ( $\mathrm{pH} \sim 8.5)$. The covalent conjugation liposomes to the ligands were carried out using cyanuric chloride (2, 4, 6 trichloro-1, 3, 5 triazine) as a coupling agent. Cyanuric chloride (CC) was reacted with the liposomes in a $1: 1$ ratio with the DSPE-PEG(2000)- $\mathrm{NH}_{2}$ for 3 hours $a t 0^{\circ} \mathrm{C}(\mathrm{pH} \sim 8.5)$. Albumin or RGD were then added dropwise to the liposomes, and the reaction was left to stir overnight at room temperature to allow the conjugation to proceed. The unconjugated moieties were then removed using Sephadex®G-100 gel filtration prepared with PBS buffer $(\mathrm{pH} \sim 7.4)$.

For estrone liposomes, we first modified the DSPEPEG(2000)- $\mathrm{NH}_{2}$. ES was reacted with $\mathrm{CC}$ in a $1: 1$ molar ratio, in the presence of two molar equivalents of triethylamine (TEA), at $0^{\circ} \mathrm{C}$ for more than three hours. The solution of estrone with TEA dissolved in dry chloroform was added dropwise to a solution of $\mathrm{CC}$ dissolved in dry chloroform at $0^{\circ} \mathrm{C}$. The functionalized ligand 2, 4 dichloro, 6 estrone-1, 3, 5 triazine (CC-ES) was then reacted with the lipids DSPEPEG(2000)- $\mathrm{NH}_{2}$ in a 1:1 molar ratio, in the presence of two equivalents of TEA using chloroform as a solvent, and the reaction was carried at $0^{\circ} \mathrm{C}$ for three hours, then the mixture was left stirring overnight at room temperature. The same synthesis scheme described above was used to conjugate the RGD and albumin moieties.

\section{B. Experimental Measurements Apparatus}

Quantifying the release of the model drug from liposomes is achieved by measuring the fluorescence change in the agent's environment. The acoustic actuation results in the release of the model drug, encapsulated inside the liposomes, and a measurable change in fluorescence is observed during this process. The percent release, $R$, of the fluorescent agent from liposomes can be computed using the following equation:

$$
R=\frac{F-F_{0}}{F_{\max }-F_{0}}
$$

where $F$ is the fluorescence intensity of the drug, $F_{0}$ is the average baseline fluorescence intensity of the solution when ultrasound is applied, and $F_{\max }$ is the fluorescence intensity of $100 \%$ drug release upon the destruction of the liposomes using the surfactant Triton X-100 .

Our experimental apparatus consisted of a low-frequency ultrasonic piezoelectric transducer to stimulate the drug release and a fluorescence measurement apparatus to quantify the release. Specifically, a 3- $\mathrm{mm}$ probe connected to a VCX 750 actuator (Vibra cells, Sonics and Material) is used to trigger drug release. The tapered probe has a water-resistant tip, and it produces a $20-\mathrm{kHz}$ frequency ultrasonic beam. The solution is placed in a cuvette with a $1 \mathrm{~cm} \times 1 \mathrm{~cm}$ opening, allowing the probe to vibrate freely which ensures maximum energy transfer into the liposomal solution. More details pertaining to the experimental apparatus can be found in [31].

The release kinetics of the chemotherapeutic agents are modeled mathematically to find the best fitting model. In total, five models are investigated. They are the zero-order, firstorder, Higuchi, Korsmeyer-Peppas, and Gompertz models. The released percent for all models is quantified as the released 
cumulative fraction of the drug and not as the absolute amount released, as seen by the fluorescence measurement equation. Release results showed that the liposomes did not release their entire drug content after exposure to ultrasound. Hence, a bias term was added to give the investigated models an extra degree of freedom to describe this behavior [32]. We treated this bias as being batch-dependent and variable, and we aim to propose an algorithm to identify this bias online to get better estimates of the model drug release.

The zero-order model assumes a constant release rate from the nanocarrier [33], given by equation (2).

$$
R_{t}=k_{0} t+b
$$

where $R_{t}$ is the fraction of drug released, $k_{0}\left[s^{-1}\right]$ is the zero-order release constant, and $b$ represents the initial release amount.

The first-order model describes the release using a firstorder differential equation [34], whose solution is given by equation (3).

$$
R_{t}=c e^{-k_{1} t}+\mathrm{b}_{1}
$$

where $R_{t}$ is the fraction of drug released at time $t, c$ is a pre-factor that depends on the initial condition, $k_{1}\left[s^{-1}\right]$ is the first-order release constant, and $b_{1}=\frac{b}{k_{1}}\left[\frac{s^{-1}}{s^{-1}}\right]$ is a term that arises from integrating the first-order model. The $b$ term represents the amount of unreleased drug amount at the end of the release process.

The Higuchi model aims to describe the drug release from a matrix system [35], given by equation (4).

$$
R_{t}=A \sqrt{D\left(2 C_{\text {lip }}-C_{s}\right) C_{s} t}
$$

where $R_{t}$ is the fraction of drug released in time $t$ per unit area $A, D$ is the diffusivity of the drug molecules in the liposome, $C_{l i p}$ is the initial drug concentration encapsulated inside the liposome, and $C_{s}$ is the drug solubility in the liposome media. The Higuchi model is simplified and rewritten as shown in equation (5).

$$
R_{t}=k_{H i} \sqrt{t}+b
$$

where $R_{t}$ is the fraction of drug released, $k_{H i}\left[s^{-1 / 2}\right]$ is the Higuchi release rate that groups all the unknowns, and $b$ is the initial fraction of the model drug released.

The Korsmeyer-Peppas model was derived from a polymeric system equation to describe drug release, and it applies to release data from several formulations of microcapsules and microspheres [36]. The power-law model is given by:

$$
R_{t}=k_{K} t^{n}+b
$$

where $R_{t}$ is the fraction of drug released in time $t, k_{H P}\left[s^{-n}\right]$ is the Korsmeyer-Peppas release rate, $n$ is the release exponent, and $b$ represents the initial release amount.

The Gompertz model [37] uses an exponential model to describe the dissolution profile, expressed by equation (7):

$$
R_{t}=1-c e^{-\alpha e^{\beta \log (t)}}+b
$$

\begin{tabular}{|c|c|c|c|c|c|c|}
\hline \multicolumn{2}{|c|}{$M S E$} & 0 -order & 1st-order & Higuchi & Korsmeyer- & Gompertz \\
\hline \multirow{3}{*}{$\begin{array}{l}\text { 罣 } \\
\text { 光 }\end{array}$} & $20 \%$ & 0.019340 & 0.000053 & 0.001845 & 0.001530 & 0.000053 \\
\hline & $25 \%$ & 0.031121 & 0.000093 & 0.004149 & 0.001907 & 0.000055 \\
\hline & $30 \%$ & 0.042181 & 0.000081 & 0.007804 & 0.002584 & 0.000055 \\
\hline \multirow{3}{*}{ 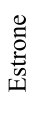 } & $20 \%$ & 0.053713 & 0.000100 & 0.014078 & 0.002628 & 0.000063 \\
\hline & $25 \%$ & 0.077735 & 0.000256 & 0.023611 & 0.002637 & 0.000117 \\
\hline & $30 \%$ & 0.072724 & 0.000124 & 0.024072 & 0.002460 & 0.000065 \\
\hline \multirow{3}{*}{ 官 } & $20 \%$ & 0.017096 & 0.000119 & 0.001587 & 0.001587 & 0.000112 \\
\hline & $25 \%$ & 0.039411 & 0.000136 & 0.006112 & 0.003347 & 0.000128 \\
\hline & $30 \%$ & 0.067389 & 0.000443 & 0.018945 & 0.005517 & 0.000424 \\
\hline \multicolumn{2}{|c|}{$\begin{array}{l}\text { Average } \\
M S E\end{array}$} & 0.046746 & 0.000156 & 0.011356 & 0.002689 & 0.000119 \\
\hline
\end{tabular}

where $R_{t}$ is the fraction of drug released at time $t, c$ is a preexponential factor that depends on the initial condition, $\alpha$ is
TABLE I

FitTing Results for Mathematical Models Across all EXPERIMENTAL RELEASE DATASETS

a scale parameter, $\beta$ is a shape parameter, and $b$ is the bias term.

To fit the experimental datasets against the proposed models, a nonlinear regression in the MATLAB was performed. In each case, bounds/limits on the parameter values were imposed such that the solution of the optimization algorithm is physically permissible. The nonlinear least-squares routine minimizes the sum of squares of the error between the measured drug release and the model-based estimate to find the best values of the model parameters (that exist within the allowable interval for each parameter). The best-fitting parameters alongside their confidence intervals are then reported, and the goodness of fit is evaluated in terms of the Mean Square Errors (MSE) between the model prediction and the experimental response. We report the results for all the models for every dataset in Table I, and we present one case of curve fitting results in Fig. 1. It is evident that models which utilize exponential functions fit the experimental data best. The first-order and the Gompertz models have low and comparableMSE, which was calculated using the five collective experimental fits. The first-order model, however, was selected due to its simple form which can be exploited in applying the Kalman filter.

\section{FILTERING}

As a consequence of the noisy nature of the release environment and the acquired measurements, we propose to enhance the best model deduced in the previous section with a stochastic filtering scheme. This filtering algorithm makes use of the dynamics model proposed, as well as a measurements model to describe the release of calcein from liposomes. Aside from the models, the algorithm also considers the existence of noises in the DDS and compensates for their existence, thus providing enhanced estimates of the dynamic states of the DDS.

The Kalman Filter (KF) is a linear Minimum Mean Square Error estimation technique that is widely applied for state estimation. Combining the structural information available from a mathematical model of the process of interest, as well as a model that describes the measurement apparatus, 

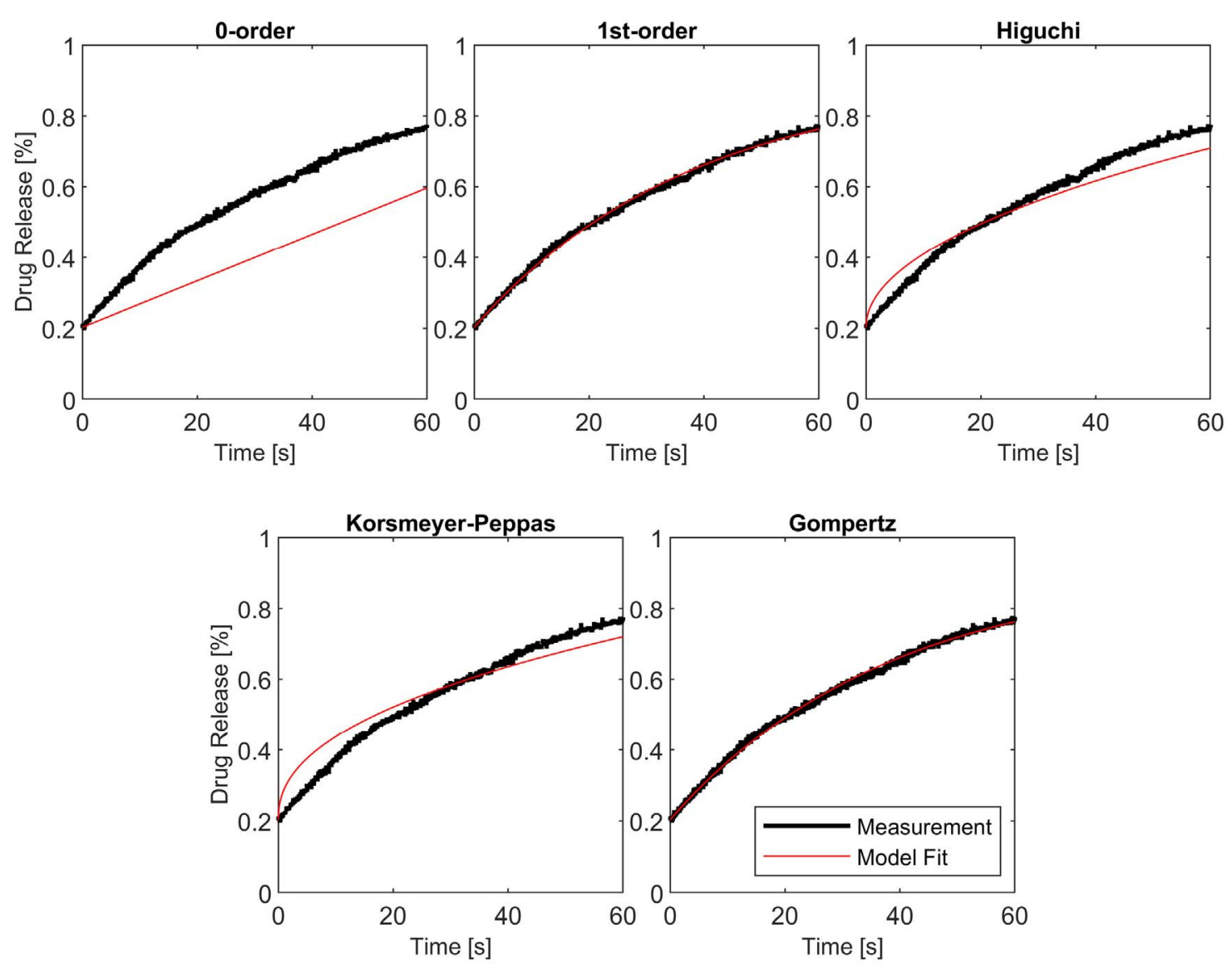

Fig. 1. Curve fitting results for the Albumin dataset at 20\% Power Density.

the $\mathrm{KF}$ is able to fuse that information together to generate optimal estimates of the states of this dynamic Drug Delivery system. However, its performance is heavily reliant on the proper tuning of the noise covariance values that are used to describe the uncertainty surrounding the measurements and dynamics of the drug release process. The linear KF is best suited to work with Gaussian noise sequences, which is the assumption made in this work.

Hence and based on previously presented results, the mathematical model chosen was the first-order bias-augmented model presented in equation (3). Taking the state vector of the DDS to include both the drug release and the bias in the release, we can write the following discrete dynamic and measurement models. The sampling frequency in all experiments was $100 \mathrm{~Hz}$.

$$
\begin{aligned}
{\left[\begin{array}{c}
R_{k+1} \\
b_{k+1}
\end{array}\right] } & =\left[\begin{array}{cc}
1-\Delta t k_{r} & \Delta t \\
0 & 1
\end{array}\right]\left[\begin{array}{l}
R_{k} \\
b_{k}
\end{array}\right]+\left[\begin{array}{ll}
1 & 0 \\
0 & 1
\end{array}\right]\left[\begin{array}{l}
w_{1 k} \\
w_{2 k}
\end{array}\right] \\
& =A x_{k}+B W_{k} \\
z_{k+1} & =\left[\begin{array}{ll}
1 & 0
\end{array}\right]\left[\begin{array}{l}
R_{k+1} \\
b_{k+1}
\end{array}\right]+v_{k+1} \\
& =C x_{k}+v_{k+1}
\end{aligned}
$$

where $R_{K}$ is the drug release state at time step $k, b_{K}$ is the bias at time step $k, \Delta t$ is the sampling time period equal to the inverse of the sampling frequency, and $w_{k}$ and $v_{k+1}$ are the dynamics and measurement noise sequences at their respective time steps. Looking at $\left(1-\Delta t k_{r}\right)$, we note that it is less than unity in magnitude, and we deduce that the above discrete model is stable. Furthermore, checking the observability, we compute a full rank Gramian, which satisfies the observability condition. The filter, then, should be able to estimate the drug release state and the bias as long as the correct model is used.

\section{A. State Estimation}

The KF algorithm is first initialized with the expected value of the DDS state vector, $x$, given the measurement, $z$, and its covariance.

$$
\begin{aligned}
E\left[x_{0} \mid z_{0}\right] & =\hat{x}_{0} \\
\operatorname{Cov}\left[x_{0} \mid z_{0}\right] & =\hat{P}_{0}
\end{aligned}
$$

The previous a posteriori state estimate, $\hat{x}_{k}$, is propagated in time to get the a priori estimate, $\bar{x}_{K+1}$, using the dynamic model. Similarly, the a priori state covariance, $\bar{P}_{K+1}$, is also realized using the previous a posteriori covariance $\hat{P}_{k}$

$$
\begin{aligned}
& \bar{x}_{k+1}=\hat{x}_{k+1 \mid k}=A \hat{x}_{k} \\
& \bar{P}_{k+1}=\hat{P}_{k+1 \mid k}=A \hat{P}_{k} A+Q
\end{aligned}
$$

The a priori measurement is then:

$$
\mathrm{E}\left[z_{k+1 \mid k}\right]=\bar{z}_{k+1}=\bar{x}_{k+1}
$$


The innovation and its covariance are defined as follows:

$$
\begin{aligned}
& \tilde{z}_{k+1}=z_{k+1}-\bar{z}_{k+1} \\
& S_{k+1}=\bar{P}_{k+1}+R
\end{aligned}
$$

The optimal Kalman gain, as well as the updated a posteriori state and covariance estimates, are then shown to be:

$$
\begin{aligned}
W_{k+1} & =\bar{P}_{k+1} S_{k+1}^{-1} \\
\hat{x}_{k+1} & =\bar{x}_{k+1}+W_{k+1} \tilde{z}_{k+1} \\
\hat{P}_{k+1} & =\bar{P}_{k+1}-W_{k+1} S_{k+1} W_{k+1}^{T}
\end{aligned}
$$

As observed from equations above, the performance of the Kalman filter, in terms of stability as well as the optimality of the estimate, is heavily reliant on the proper selection of the noise covariance magnitudes, $Q$ and $R$. If the covariance magnitudes are not correct in representing the true uncertainty in the dynamic and measurement models, the estimates will not be optimal. This will then reflect on the accuracy of the estimate. The next section addresses this optimality issue; we propose an online real-time noise covariance estimation algorithm to tune the covariance magnitudes to be representative of the true statistics of the noise sequences.

\section{B. Online Uncertainty Identification Algorithm}

It is of interest to invoke the adaptation in the KF algorithm such that the dynamics and measurements covariance magnitudes used by the KF are as close to the real magnitudes as possible, hence the filter estimates are as close to optimal as possible. Here, we describe and implement an adaptive $\mathrm{KF}$ (AKF) approach based on covariance matching. This approach exploits the theoretical definitions of the innovation, which is defined as the difference between the acquired measurement and the predicted measurement value, and the residual, defined as the difference between the acquired measurement and the updated measurement value.

At time step $k+1$, the innovation, $\tilde{z}_{k+1}$, and the residual, $\epsilon_{k+1}$, are given by:

$$
\begin{aligned}
\tilde{z}_{k+1} & =z_{k+1}-\bar{z}_{k+1} \\
& =z_{k+1}-H \bar{x}_{k+1} \\
\epsilon_{k+1} & =z_{k+1}-H \hat{x}_{k+1}
\end{aligned}
$$

From the KF equations, the measurement covariance estimate can be written as:

$$
\hat{R}_{k}=S_{k+1}-H \bar{P}_{k+1} H^{T}
$$

This estimate is the difference between two positive definite matrices, which can prove problematic as it does not ensure the positive definiteness of the covariance matrix. Consequently, following Wang [38], a residual-based approach is to be followed.

Applying the error propagation law to the residual equation in equation IV gives:

$$
\begin{aligned}
\operatorname{Cov}\left(\epsilon_{k+1}\right) & =\operatorname{Cov}\left(z_{k+1}-H \hat{x}_{k+1}\right) \\
& =\operatorname{Cov}\left(H x_{k+1}+v_{k+1}-H \hat{x}_{k+1}\right) \\
& =\operatorname{Cov}\left(v_{k+1}\right)-H \operatorname{Cov}\left(x_{k+1}-\hat{x}_{k+1}\right) H^{T} \\
& =R_{k+1}-H \hat{P}_{k+1} H^{T}
\end{aligned}
$$
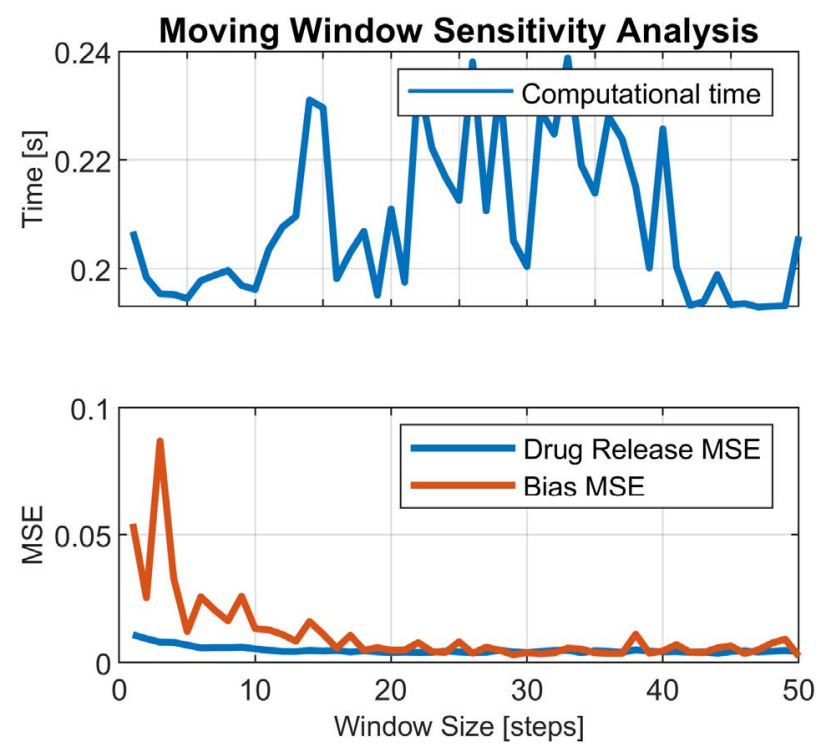

Fig. 2. Moving Window Step Size Sensitivity Analysis.

The covariance of the filtering residuals can be approximated in a moving window of $n$ measurements to be

$$
\operatorname{Cov}\left(\epsilon_{k+1}\right)=\frac{1}{n} \sum_{i=0}^{n-1} \epsilon_{k+1-i} \epsilon_{k+1-i}^{T}
$$

Alternatively, one could use a forgetting factor, $\alpha$, to modify the above approach to invoke a more tunable adaptation from the estimation algorithm at a lower computational burden [25]. A higher value of $\alpha$ will put more weight on previous estimates and will cause a slower and more stable adaptation of the covariance estimate. This can be written as follows:

$$
\hat{R}_{k+1}=\alpha R_{k}+(1-\alpha)\left[\epsilon_{k+1} \epsilon_{k+1}^{T}+H \hat{P}_{k+1} H^{T}\right]
$$

Testing the two approaches, however, showed slightly better performance with the moving window-based approach. As a consequence of the moving window averaging not posing a significant computational burden, it will be used in favor of the forgetting factor approach.

The covariance estimate of the dynamics noise can be written, for the DDS dynamics, as:

$$
\begin{aligned}
\hat{w}_{k} & =\hat{x}_{k+1}-A x_{k} \\
& =\hat{x}_{k+1}-\bar{x}_{k+1} \\
& =W_{k+1} \tilde{z}_{k+1} \\
& =W_{k+1}\left(z_{k+1}-H \bar{x}_{k+1}\right)
\end{aligned}
$$

Applying the error propagation law to the above equation yields:

$$
\begin{aligned}
\operatorname{Cov}\left(\hat{w}_{k}\right) & =\operatorname{Cov}\left(W_{k+1} \tilde{z}_{k+1}\right) \\
& =W_{k+1} \operatorname{Cov}\left(\tilde{z}_{k+1}\right) W_{k+1}^{T} \\
\hat{Q}_{k} & =W_{k+1} \frac{1}{n} \sum_{i=0}^{n-1} \tilde{z}_{k+1-i} \tilde{z}_{k+1-i}^{T} W_{k+1}^{T}
\end{aligned}
$$



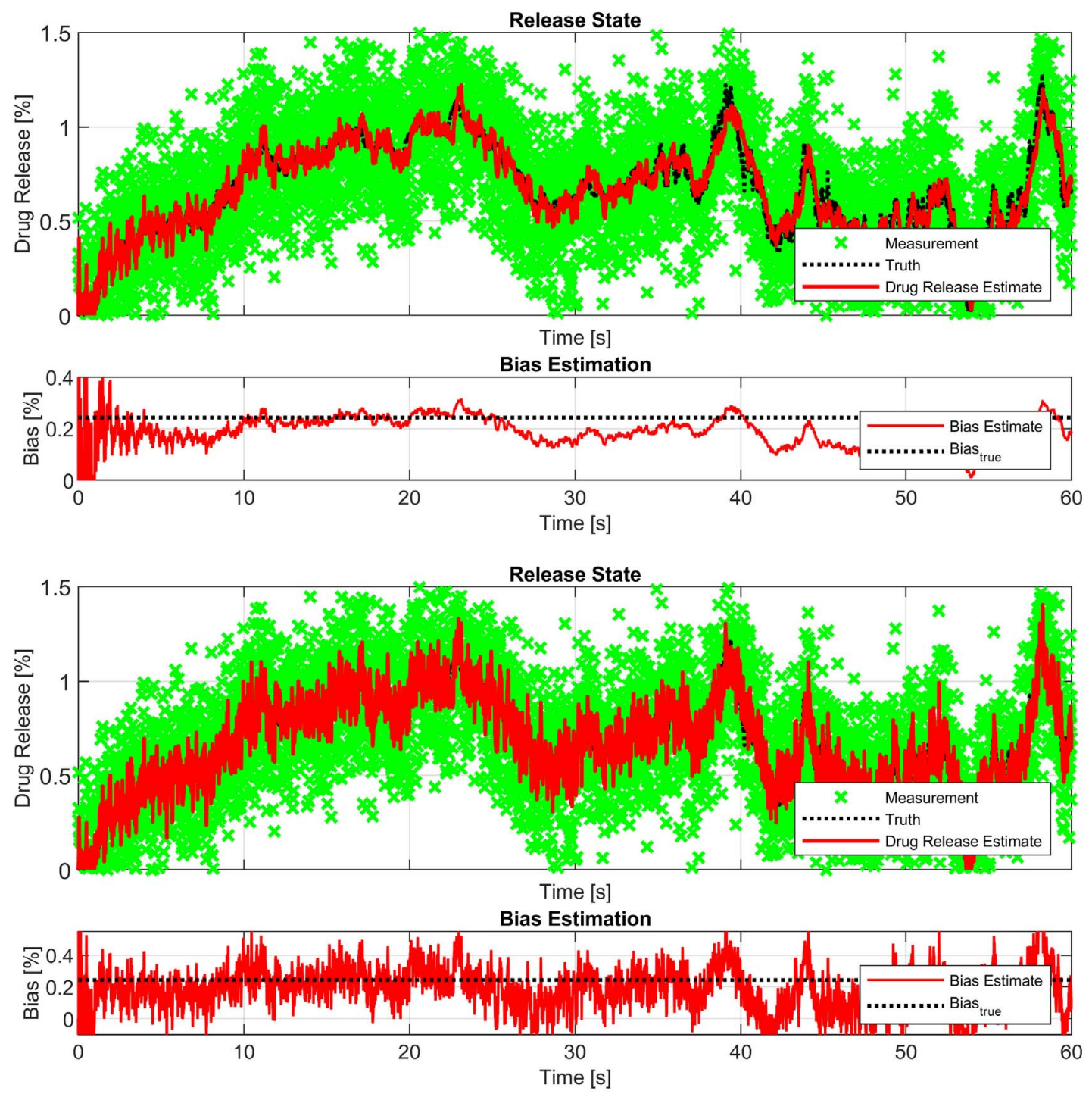

Fig. 3. Algorithm performance in the simulated environment Top figure displays the Adaptive Kalman Filter, and the bottom one displays the Classic Kalman Filter.

Applying the forgetting factor methodology, the dynamics noise covariance estimator becomes:

$$
\begin{aligned}
\hat{Q}_{k+1} & =\alpha Q_{k}+ \\
& =(1-\alpha)\left[W_{k+1} \tilde{z}_{k+1} \tilde{z}_{k+1}^{T} W_{k+1}^{T}\right]
\end{aligned}
$$

As with the measurement noise covariance estimation, moving window averaging will be employed to approximate the covariance.

The next section will implement the proposed DDS modeling algorithm and extensively test it out in both simulated and experimental settings.

\section{Results}

It is imperative to deploy the proposed modeling algorithm in a simulated environment to test and validate its performance against a known truth. First, the size of the moving window that approximates the covariances in the adaptive algorithm was investigated in a sensitivity analysis study.
Then, a simulated study case was designed to stress test the proposed algorithm. Finally, the algorithm was used to process experimental data after the validation in our simulated setting.

\section{A. Moving Window Sensitivity Analysis}

The moving window approach used to approximate covariances operated on a predefined number of points, and it averages those points to approximate the covariance. It is of interest to study the effect of the size of the moving window on both the performance of the filtering algorithm and the computational burden that the size of the window imparts on the machine running the algorithm.

To identify a suitable window size, Monte Carlo simulations were performed by investigating moving window sizes that range from 1 to 50. We ran each simulation 50 times, and the Mean Square Errors (MSE), as well as the computational time needed to execute the code, were recorded. The MSE is 

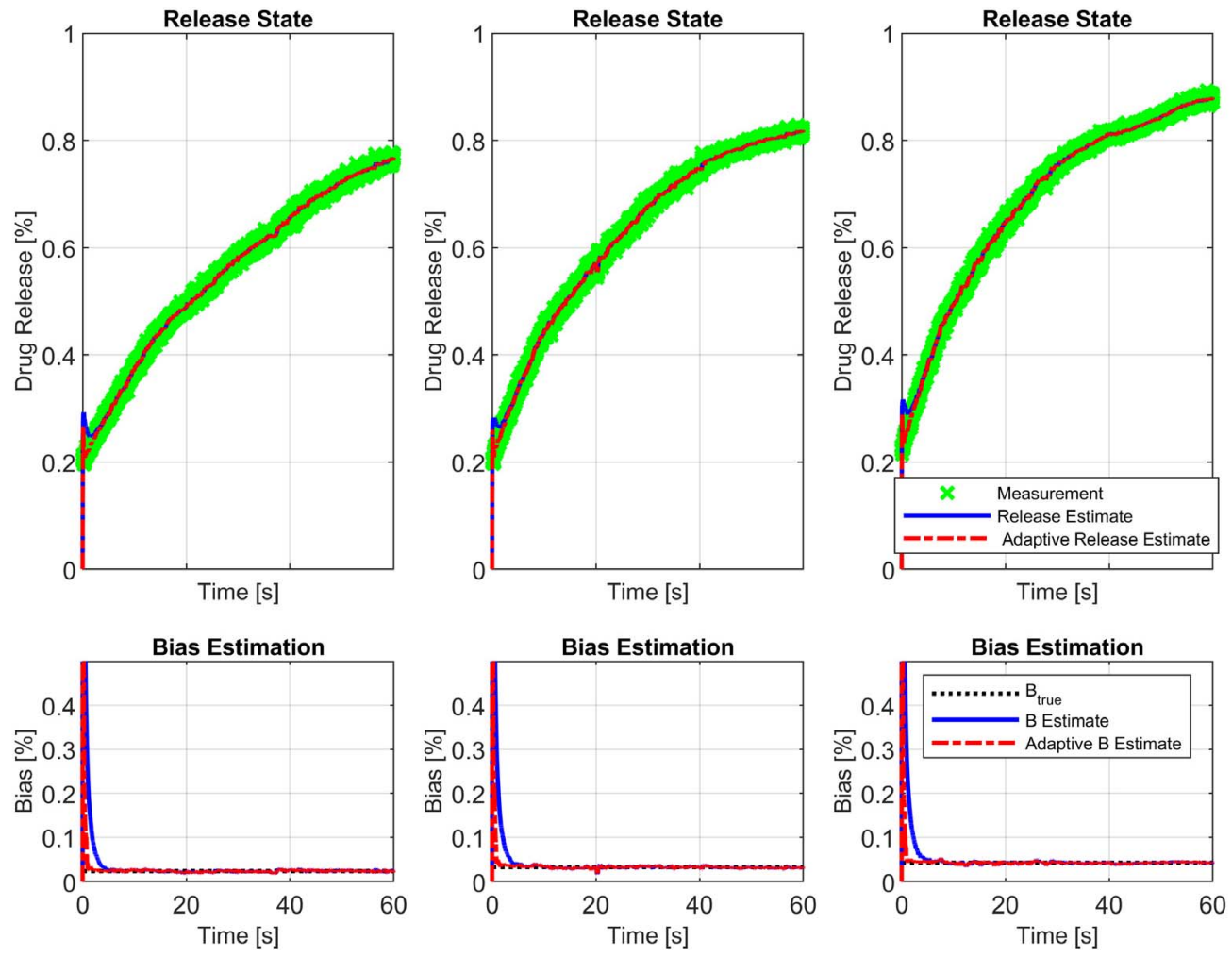

Fig. 4. Filtering performance on the Albumin dataset at 7.46, 9.85, and $17.31 \frac{\mathrm{mW}}{\mathrm{cm}^{2}}$ US power densities (from left to right).

given by:

$$
\operatorname{MSE}=\frac{1}{K} \sum_{i=1}^{K}\left(x_{i}-\hat{x}_{i}\right)^{2}
$$

where $K$ is the length of the measurements window and $x_{i}-\hat{x}_{i}$ is the error between the true state and the estimate.

Figure 2 presents the Monte Carlo simulation averaged results. As the window size increases, a sharp drop in the MSE is observed with the bias state, which shows the most significant change. After approximately20 steps, the MSE stabilizes to almost constant levels. Similarly, the drug release state MSE drops to constant levels, but the change is small in magnitude when compared to the bias. The computational performance of the algorithm displays an erratic trend, but it seems to hover around an average value of $0.22 s$ to process the entire 6000 data points. Therefore, we chose the moving window size of 20 steps to execute fewer computations than deemed necessary.

\section{B. Simulation Results}

The algorithms were implemented in an exaggerated noisy environment for rigorous testing purposes. To evaluate the performance and robustness of the adaptation algorithm, a change in the noise sequences being simulated was induced. For the first 35 seconds, the noise covariance magnitudes were $Q=5 \times 10^{-5}$ and $R=5 \times 10^{-3}$, and they suddenly change to $Q=5 \times 10^{-4}$ and $R=5 \times 10^{-2}$ for the rest of the simulated time. This simulates a catastrophic degradation in the data acquisition apparatus accompanied by a change in the
TABLE II

Simulated EnVironment Filter Performance

\begin{tabular}{lcc}
\hline \hline Estimated State & AKF MSE & KF MSE \\
\hline Drug Release State & 0.0042 & 0.0086 \\
Bias Estimate State & 0.0115 & 0.0231 \\
\hline \hline
\end{tabular}

$$
b=0.242
$$

dynamics of the DDS. The simulated test case is designed to stress test the algorithms in order to showcase the benefits of the proposed adaptive approach.

The DDS parameters, as well as the AKF initialization used to simulate the response of the DDS, are as follows:

$$
\begin{aligned}
\hat{x}_{0} & =\bar{x}_{0}=\left[\begin{array}{l}
0 \\
\epsilon
\end{array}\right] \\
\hat{P}_{0} & =\bar{P}_{0}=\left[\begin{array}{cc}
\epsilon & 0 \\
0 & 10
\end{array}\right] \\
W_{0} & =\left[\begin{array}{l}
1 \\
1
\end{array}\right] \\
Q_{0} & =\left[\begin{array}{ll}
1 & 0 \\
0 & 1
\end{array}\right] \times 5 \times 10^{-5} \\
R_{0} & =5 \times 10^{-3} \\
k_{r} & =0.259 \\
b & =0.242
\end{aligned}
$$

The simulation results for a non-optimal Kalman Filter are presented alongside the results of the proposed Adaptive Kalman Filter in Fig. 3. The acquired measurements are 

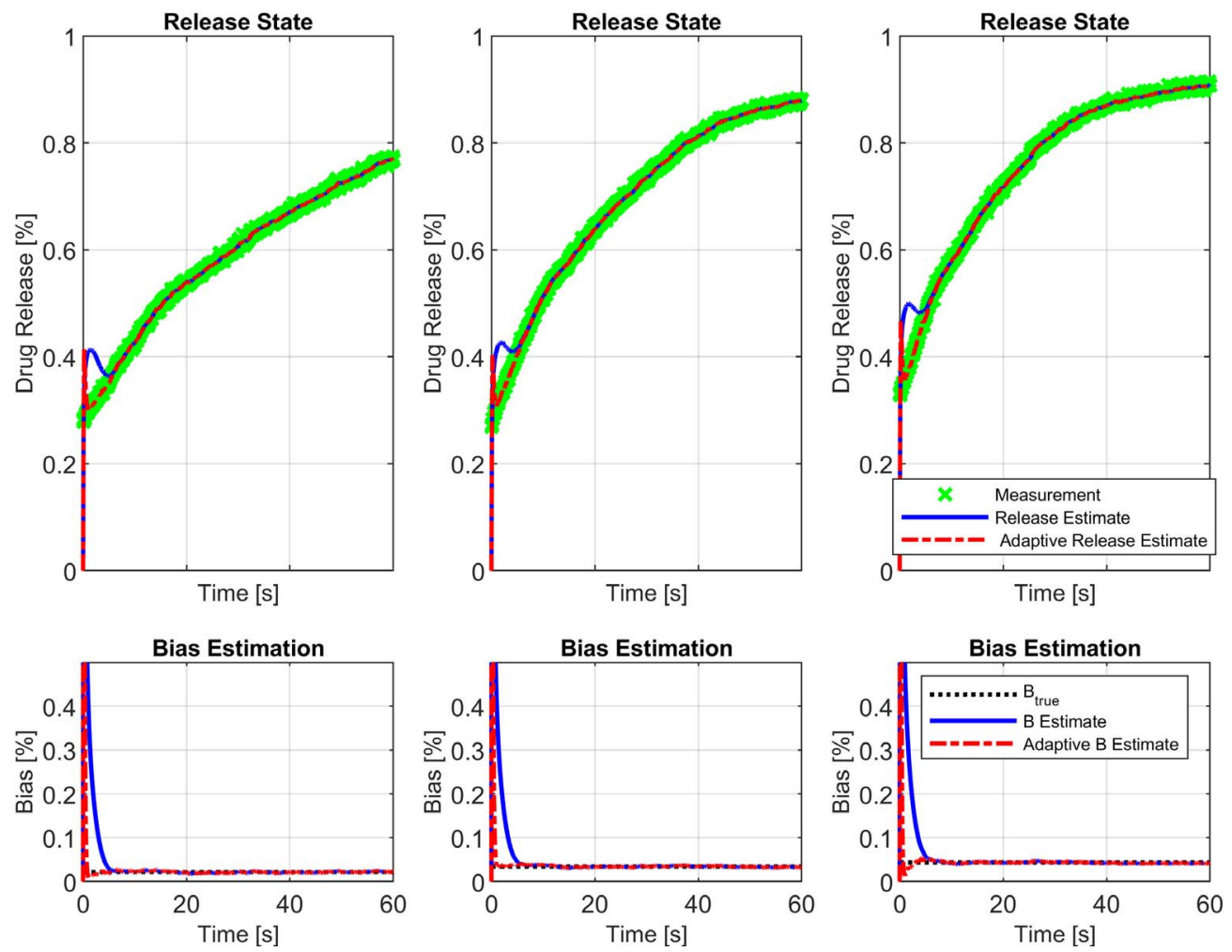

Fig. 5. Filtering performance on the Estrone dataset at 7.46, 9.85, and $17.31 \frac{\mathrm{mW}}{\mathrm{cm}^{2}}$ US power densities (from left to right).
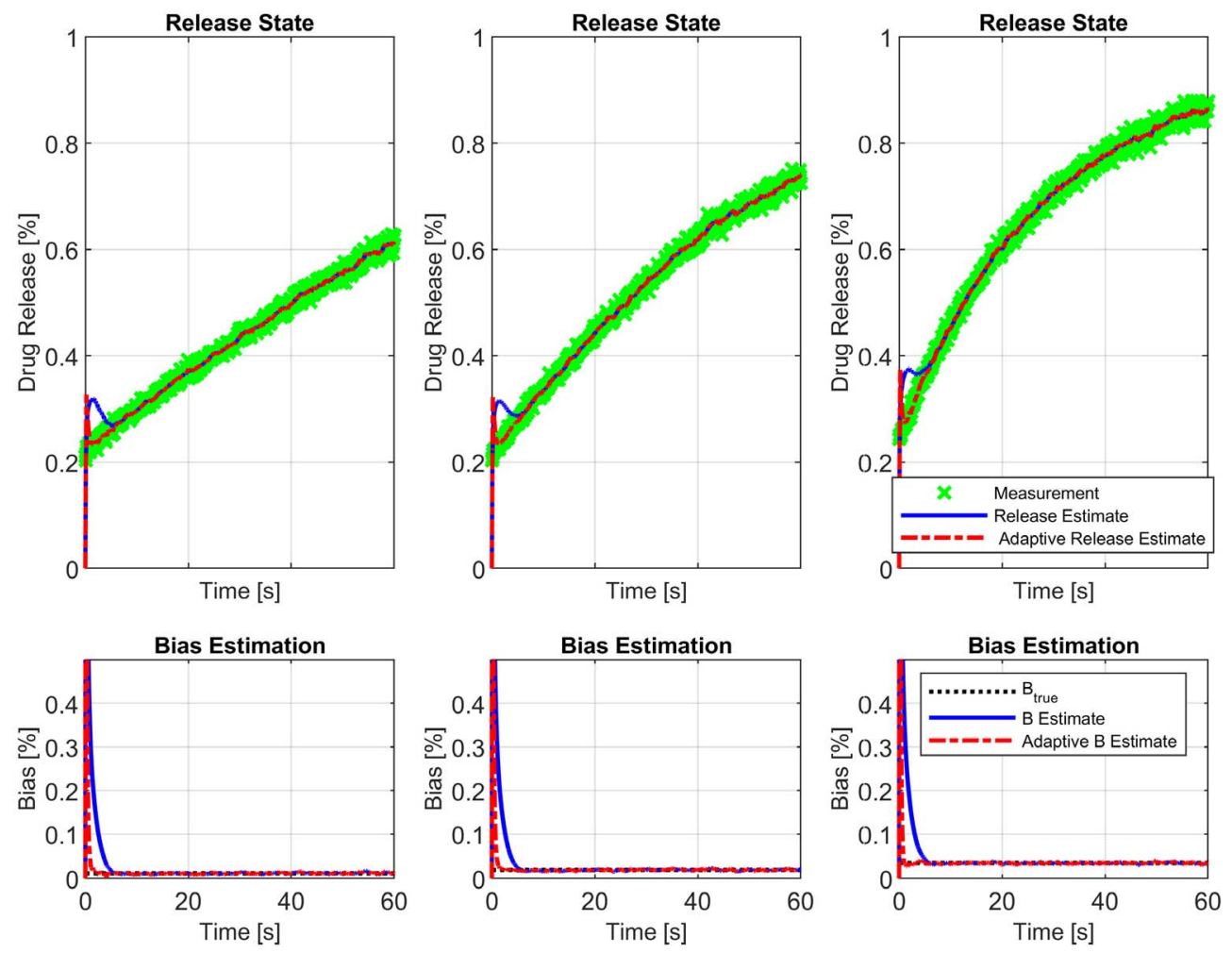

Fig. 6. Filtering performance on the RGD dataset at 7.46,9.85, and $17.31 \frac{\mathrm{mW}}{\mathrm{cm}^{2}}$ US power densities (from left to right).

extremely noisy and are scattered around the true state of drug release. They are not representative of the true state of drug release. It is evident that the filter quickly adapts at the beginning to track the true states of drug release, as well as the bias. When the simulated change in noise covariance magnitudes happens, the filter again proves its robustness and 
TABLE III

EXPERIMENTAL AKF PERFORMANCE

\begin{tabular}{ccccccc}
\hline \hline \multirow{2}{*}{$\begin{array}{c}\text { Experimental AKF } \\
\text { MSE }\end{array}$} & \multicolumn{2}{c}{ Albumin dataset } & \multicolumn{2}{c}{ Estrone dataset } & \multicolumn{2}{c}{ RGD dataset } \\
\cline { 2 - 6 } Release & Bias & $\begin{array}{c}\text { Drug } \\
\text { Release }\end{array}$ & Bias & $\begin{array}{c}\text { Drug } \\
\text { Release }\end{array}$ & Bias \\
\hline $\begin{array}{l}\text { Experiment 1 } \\
\left(7.46 \frac{\mathrm{mW}}{\mathrm{cm}}\right)\end{array}$ & $2.818 \times 10^{-5}$ & $4.515 \times 10^{-6}$ & $1.333 \times 10^{-5}$ & $7.806 \times 10^{-7}$ & $1.108 \times 10^{-4}$ & $6.356 \times 10^{-7}$ \\
$\quad \begin{array}{l}\text { Experiment } 2 \\
\left(9.85 \frac{\mathrm{mW}}{\mathrm{cm}}\right)\end{array}$ & $3.281 \times 10^{-5}$ & $3.371 \times 10^{-6}$ & $1.389 \times 10^{-5}$ & $7.124 \times 10^{-7}$ & $1.210 \times 10^{-4}$ & $6.019 \times 10^{-7}$ \\
$\begin{array}{l}\text { Experiment } 3 \\
\left(17.31 \frac{\mathrm{mW}}{\mathrm{cm}^{2}}\right)\end{array}$ & $3.181 \times 10^{-5}$ & $4.530 \times 10^{-6}$ & $1.299 \times 10^{-5}$ & $1.093 \times 10^{-7}$ & $1.386 \times 10^{-4}$ & $1.403 \times 10^{-6}$ \\
\hline \hline
\end{tabular}

TABLE IV

EXPERIMENTAL KF PERFORMANCE

\begin{tabular}{|c|c|c|c|c|c|c|}
\hline \multirow{2}{*}{$\begin{array}{l}\text { Experimental KF } \\
\text { MSE }\end{array}$} & \multicolumn{2}{|c|}{ Albumin dataset } & \multicolumn{2}{|c|}{ Estrone dataset } & \multicolumn{2}{|c|}{ RGD dataset } \\
\hline & $\begin{array}{r}\text { Drug } \\
\text { Release }\end{array}$ & Bias & $\begin{array}{r}\text { Drug } \\
\text { Release }\end{array}$ & Bias & $\begin{array}{r}\text { Drug } \\
\text { Release }\end{array}$ & Bias \\
\hline $\begin{array}{l}\text { Experiment } 1 \\
\left(7.46 \frac{\mathrm{mW}}{\mathrm{cm}^{2}}\right)\end{array}$ & $4.615 \times 10^{-5}$ & $1.202 \times 10^{-3}$ & $3.674 \times 10^{-5}$ & $5.177 \times 10^{-5}$ & $1.411 \times 10^{-4}$ & $5.799 \times 10^{-5}$ \\
\hline $\begin{array}{l}\text { Experiment } 2 \\
\left(9.85 \frac{\mathrm{mW}}{\mathrm{cm}^{2}}\right)\end{array}$ & $4.969 \times 10^{-5}$ & $1.238 \times 10^{-3}$ & $3.547 \times 10^{-5}$ & $5.264 \times 10^{-5}$ & $1.486 \times 10^{-4}$ & $5.370 \times 10^{-5}$ \\
\hline $\begin{array}{l}\text { Experiment } 3 \\
\left(17.31 \frac{\mathrm{mW}}{\mathrm{cm}^{2}}\right)\end{array}$ & $5.338 \times 10^{-5}$ & $1.469 \times 10^{-3}$ & $4.177 \times 10^{-5}$ & $7.100 \times 10^{-5}$ & $1.711 \times 10^{-4}$ & $7.013 \times 10^{-5}$ \\
\hline
\end{tabular}

tracks the erratic response of the DDS states. Some oscillations are still visible, especially with the bias state, (note the high level of noise injected into the processes).

Table II documents the calculated MSE for the AKF and the KF. The MSE between the true state of drug release and the AKF estimate was computed to be $M S E_{R}=0.0042$, while the MSE for the bias estimate was computed to be $M S E_{b}=0.0115$. On the other hand, the MSE between the true state of drug release and the KF estimate was computed to be $M S E_{R}=0.0086$, while the MSE for the bias estimate was computed to be $M S E_{b}=0.0231$. The performance of the AKF is clearly superior in the simulated setting, and it warrants deploying the algorithm in an experimental setting.

\section{EXPERIMENTAL RESULTS}

Now that the AKF performed well in the simulated environment, we deploy it in an experimental setting on the acquired drug releases discussed earlier. The releases of calcein from three types of targeted nanocarriers were acquired at the three distinct US power densities of 7.46, 9.85, and $17.31 \frac{\mathrm{mW}}{\mathrm{cm}^{2}}$, which are equivalent to $20 \%, 25 \%$, and $30 \%$ of the total available power, respectively.

Fig. 4, 5, and 6 display the performance of both the KF and the AKF on the acquired experimental datasets. The levels of measurements noise are clearly not as large as those in the simulated environment, but they are scattered around the estimate and not very smooth in nature. This noise is mainly attributed to the nature of US. Nonetheless, the AKF converges very quickly to the true bias in comparison to the KF. The true state of bias was identified through the postprocessing of the data through the curve fitting of the model. In turn, identifying the true bias very early throughout the release experiment results in the filter producing estimates that are not as scattered as the measurement points. Moreover, the AKF tracks the measurements much better than the KF. As the true state of drug release is unknown, the MSE statistics presented in this work are computed between the measurements and the filter estimates. It is of interest, however, to stress on the filter's capacity to quickly converge on the true bias, which gives merit to the drug release estimate being also close to the true state of drug release and our proposed approach. Table III documents the experimental MSE for the AKF and Table IV documents the experimental MSE for the KF. In all cases, the MSE with the AKF is slightly better to significantly better than its nonadaptive counterpart. In comparison to the KF, the AKF MSE difference is as small as being half the KF and as large as being three orders of magnitude less than the KF. The proposed algorithm consistently performs better than the classic one.

\section{Discussion AND CONCLUSION}

Reliable prediction of the dynamic behavior of chemotherapeutic DDSs is essential to reaching a selective treatment system where the drug delivery is controlled using algorithms 
such as Model Predictive Controller (MPC). This type of intelligent control requires a robust mathematical model and quality measurements of the amount of drug delivered to the patient. This is where our proposed modeling approach can be deployed; the AKF fuses the structural information of a firstorder kinetics model that is capable of describing the release process with the filtering scheme that is able to estimate the state of drug release regardless of the quality of measurements acquired. The need for the proposed adaptation arises because the noise uncertainty structure is inherently unknown and is difficult to know beforehand.

Numerous mathematical models reported in literature were first studied to determine the best fit to the experimental data. Then, an adaptive variant of a Kalman filter was designed to make use of the dynamics and measurements models, while adaptively estimating the noise covariance magnitudes plaguing the dynamics and measurement processes. The proposed approach was deployed in a simulated environment where it performed exceptionally well compared to the classical filter. Afterwards, the algorithm was used to filter the experimental data, identify the true state of bias in the process and give accurate drug release estimates.

The next step is to study the relation between ultrasonic power density and the kinetic release constants at play in this triggered DDS. This relationship can in turn be used to design an MPC system capable of controlling drug delivery in time and space both theoretically and experimentally.

\section{REFERENCES}

[1] Y. Pommier, "DNA topoisomerase I and II in cancer chemotherapy: Update and perspectives," Cancer Chemother. Pharmacol., vol. 32, no. 2, pp. 103-108, Mar. 1993.

[2] G. J. Peters, C. L. van der Wilt, C. J. van Moorsel, J. R. Kroep, A. M. Bergman, and S. P. Ackland, "Basis for effective combination cancer chemotherapy with antimetabolites," Pharmacol. Therapeutics, vol. 87, nos. 2-3, pp. 227-253, 2000.

[3] E. K. Rowinsky, "The development and clinical utility of the taxane class of antimicrotubule chemotherapy agents," Annu. Rev. Med., vol. 48, no. 1, pp. 353-374, Feb. 1997.

[4] A. Schroeder et al., "Controlling liposomal drug release with low frequency ultrasound: Mechanism and feasibility," Langmuir, vol. 23, no. 7, pp. 4019-4025, Mar. 2007.

[5] M. T. Larsen, M. Kuhlmann, M. L. Hvam, and K. A. Howard, "Albuminbased drug delivery: Harnessing nature to cure disease," Mol. Cellular Therapies, vol. 4, no. 1, p. 3, Dec. 2016

[6] S. Rai et al., "Targeted delivery of doxorubicin via estrone-appended liposomes," J. Drug Targeting, vol. 16, no. 6, pp. 455-463, Jan. 2008.

[7] Y. Sun, C. Kang, F. Liu, Y. Zhou, L. Luo, and H. Qiao, "RGD peptidebased target drug delivery of doxorubicin nanomedicine," Drug Dev. Res., vol. 78, no. 6, pp. 283-291, Sep. 2017.

[8] D. Y. Arifin, L. Y. Lee, and C.-H. Wang, "Mathematical modeling and simulation of drug release from microspheres: Implications to drug delivery systems," Adv. Drug Del. Rev., vol. 58, nos. 12-13, pp. 1274-1325, Nov. 2006.

[9] J. Siepmann and F. Siepmann, "Mathematical modeling of drug delivery," Int. J. Pharmaceutics, vol. 364, no. 2, pp. 328-343, Dec. 2008.

[10] G. A. Husseini, F. S. Mjalli, W. G. Pitt, and N. M. Abdel-Jabbar, "Using artificial neural networks and model predictive control to optimize acoustically assisted Doxorubicin release from polymeric micelles," Technol. Cancer Res. Treatment, vol. 8, no. 6, pp. 479-488, 2009.

[11] S. Dash, P. N. Murthy, L. Nath, and P. Chowdhury, "Kinetic modeling on drug release from controlled drug delivery systems," Acta Pol. Pharmaceutics, vol. 67, no. 3, pp. 217-223, May 2010.

[12] A. Abusara, M. Abdel-Hafez, and G. Husseini, "Measuring the acoustic release of a chemotherapeutic agent from folate-targeted polymeric micelles," J. Nanosci. Nanotechnol., vol. 18, no. 8, pp. 5511-5519, Aug. 2018.
[13] M. Abdel-Hafez and G. A. Husseini, "Predicting the release of chemotherapeutics from the core of polymeric micelles using ultrasound," IEEE Trans. Nanobiosci., vol. 14, no. 4, pp. 378-384, Jun. 2015.

[14] A. Wadi, M. Abdel-Hafez, and G. A. Husseini, "Modeling and biasrobust estimation of the acoustic release of chemotherapeutics from liposomes," J. Biomed. Nanotechnol., vol. 15, no. 1, pp. 162-169, Jan. 2019.

[15] D. Fey, R. Findeisen, and E. Bullinger, "Parameter estimation in kinetic reaction models using nonlinear observers facilitated by model extensions," IFAC Proc. Volumes, vol. 41, no. 2, pp. 313-318, 2008.

[16] G. Lillacci and P. Valigi, "State estimation for a model of gene expression," in Proc. IEEE Int. Symp. Circuits Syst., May 2008, pp. 2046-2049.

[17] C. H. Wells, "Application of modern estimation and identification techniques to chemical processes," AIChE J., vol. 17, no. 4, pp. 966-973, Jul. 1971.

[18] D. Dochain, "State and parameter estimation in chemical and biochemical processes: A tutorial," J. Process Control, vol. 13, no. 8, pp. 801-818, Dec. 2003.

[19] Z. Wang, X. Liu, Y. Liu, J. Liang, and V. Vinciotti, "An extended Kalman filtering approach to modeling nonlinear dynamic gene regulatory networks via short gene expression time series," IEEE/ACM Trans. Comput. Biol. Bioinformatics, vol. 6, no. 3, pp. 410-419, Jul. 2009.

[20] G. Lillacci and M. Khammash, "Parameter estimation and model selection in computational biology," PLoS Comput. Biol., vol. 6, no. 3, Mar. 2010, Art. no. e1000696.

[21] R. N. Gutenkunst, J. J. Waterfall, F. P. Casey, K. S. Brown, C. R. Myers, and J. P. Sethna, "Universally sloppy parameter sensitivities in systems biology models," PLoS Comput. Biol., vol. 3, no. 10, p. e189, Oct. 2007.

[22] A. Wadi, M. Abdel-Hafez, and G. A. Husseini, "Identification of the uncertainty structure to estimate the acoustic release of chemotherapeutics from polymeric micelles," IEEE Trans. Nanobiosci., vol. 16, no. 7, pp. 609-617, Oct. 2017.

[23] B. Gao, S. Gao, G. Hu, Y. Zhong, and C. Gu, "Maximum likelihood principle and moving horizon estimation based adaptive unscented Kalman filter," Aerosp. Sci. Technol., vol. 73, pp. 184-196, Feb. 2018.

[24] Z. Gao, D. Mu, S. Gao, Y. Zhong, and C. Gu, "Adaptive unscented Kalman filter based on maximum posterior and random weighting," Aerosp. Sci. Technol., vol. 71, pp. 12-24, Dec. 2017.

[25] S. Akhlaghi, N. Zhou, and Z. Huang, "Adaptive adjustment of noise covariance in Kalman filter for dynamic state estimation," in Proc. IEEE Power Energy Soc. Gen. Meeting, Jul. 2017, pp. 1-5.

[26] S. C. Rutan, "Adaptive Kalman filtering," Anal. Chem., vol. 63, no. 22, pp. 1103A-1109A, Nov. 1991.

[27] M. A. Zagrobelny and J. B. Rawlings, "Identifying the uncertainty structure using maximum likelihood estimation," in Proc. Amer. Control Conf. (ACC), Jul. 2015, pp. 422-427.

[28] N. M. Salkho et al., "Ultrasonically controlled estrone-modified liposomes for estrogen-positive breast cancer therapy," Artif. Cells, Nanomed., Biotechnol., vol. 46, no. 2, pp. 462-472, Apr. 2018.

[29] M. Mahmoud, The Effect of Ultrasound on the Drug Delivery of RGD-Targeted Liposomes. Sharjah, United Arab Emirates: Amer. Univ. Sharjah, 2018.

[30] R. Turki, Preparation of Albumin-Targeted Liposomes and the Study of Their Release Characteristics Using Ultrasound. Sharjah, United Arab Emirates: Amer. Univ. Sharjah, 2016.

[31] S. E. Ahmed, H. G. Moussa, A. M. Martins, M. H. Al-Sayah, and G. A. Husseini, "Effect of $\mathrm{pH}$, ultrasound frequency and power density on the release of calcein from stealth liposomes," Eur. J. Nanomed., vol. 8, no. 1, pp. 31-43, 2016.

[32] Y. Bar-Shalom and X.-R. Li, Estimation with Applications to Tracking and Navigation. Hoboken, NJ, USA: Wiley, 2001.

[33] L. Yang and R. Fassihi, "Zero-order release kinetics from a selfcorrecting floatable asymmetric configuration drug delivery system," J. Pharmaceutical Sci., vol. 85, no. 2, pp. 170-173, Feb. 1996.

[34] G. S. Banker and C. T. Rhodes, Modern Pharmaceutics. New York, NY, USA: Marcel Dekker, 2002.

[35] T. Higuchi, "Mechanism of sustained-action medication. Theoretical analysis of rate of release of solid drugs dispersed in solid matrices," J. Pharmaceutical Sci., vol. 52, no. 12, pp. 1145-1149, Dec. 1963.

[36] R. W. Korsmeyer, R. Gurny, E. Doelker, P. Buri, and N. A. Peppas, "Mechanisms of solute release from porous hydrophilic polymers," Int. J. Pharmaceutics, vol. 15, no. 1, pp. 25-35, May 1983.

[37] S. C. Chow, Encyclopedia of Biopharmaceutical Statistics. Boca Raton, FL, USA: CRC Press, 2018.

[38] J. Wang, "Stochastic modeling for real-time kinematic GPS/GLONASS positioning," Navigation, vol. 46, no. 4, pp. 297-305, 1999. 